\title{
Interaction of electrical and ultrasonic neuromodulation: a computational study
}

\section{Thomas Tarnaud, Wout Joseph, Luc Martens, Timothy Van Renterghem, Emmeric Tanghe}

Introduction: In the last decade, transcranial focused ultrasound (tFUS) has gained significant attention as a technique to modulate neuronal activity reversibly, non-invasively and with high spatial accuracy. Combining tFUS with existing electrical neurostimulation modalities (tDCS, TMS, DBS...) might help to unravel the underlying biophysical and physiological mechanisms of ultrasonic neuromodulation. E.g., in a recent study of Legon et al. (2018) tFUS is paired with TMS, allowing to assess the effect of ultrasound on TMS metrics (MEP, SICl, ICF...). In this computational study, we investigate the interaction of ultrasonic and electrical neuromodulation in the neuronal intramembrane cavitation excitation model (NICE; Plaksin et al. $(2014,2016))$.

Methods: The bilayer sonophore model (Krasovitski et al. (2011)) is used to compute the neuronal response to simultaneous electrical and ultrasonic stimulation for cortical (regular spiking (RS), fast spiking (FS), and low-threshold spiking (LTS); Pospischil-models) and subcortical (subthalamic nucleus (STN); Otsuka-model) neurons. Interaction of ultrasound and electrical current with continuous-wave and pulsed waveforms is considered.

Results: Excitation thresholds and firing rate contours are determined by interactions between the electrical injected current and the ultrasonic wave. E.g., simultaneous application of subthreshold electrical current and subthreshold ultrasonic intensity is capable of eliciting spikes, due to interaction of both stimulation modalities. Interestingly, the order of excitability of the computational models can be reversed by combining hyperpolarizing (negative) electrical currents with ultrasound.

Conclusion: In this study, the interaction of simultaneously applied electrical and ultrasonic waveforms in the NICE-model is investigated. Our results indicate that combining both stimulation modalities might improve safety (lower threshold intensities per modality), spatial accuracy and selectivity (manipulation of the order of excitability). As future work, we intend to investigate the effects of simultaneous application of ultrasound and electrical neuromodulation on computational network models. 